\title{
Zinc status, insulin resistance and glycoxidative stress in elderly subjects with type 2 diabetes mellitus
}

\author{
DANIELA GRĂDINARU ${ }^{1 *}$, DENISA MARGINĂ ${ }^{1 *}$, ANCA UNGURIANU $^{1}$, \\ GEORGIANA NIȚULESCU ${ }^{2}$, CĂTĂLINA MONICA PENA ${ }^{3}$, \\ CONSTANTIN IONESCU-TÎRGOVIȘTE ${ }^{4}$ and RUCSANDRA DĂNCIULESCU MIULESCU ${ }^{4}$ \\ Departments of ${ }^{1}$ Biochemistry and ${ }^{2}$ Pharmaceutical Technology, Faculty of Pharmacy, \\ 'Carol Davila' University of Medicine and Pharmacy, 020956 Bucharest; \\ ${ }^{3}$ Biology of Aging Department, 'Ana Aslan' National Institute of Gerontology and Geriatrics, \\ 011241 Bucharest; ${ }^{4}$ Clinical Department, 'Nicolae Paulescu' Institute of Diabetes, \\ Metabolic and Nutrition Diseases, 020475 Bucharest, Romania
}

Received August 6, 2021; Accepted September 6, 2021

DOI: $10.3892 / \mathrm{etm} .2021 .10829$

\begin{abstract}
Zinc deficiencies have been reported in numerous pathologies, such as diabetes mellitus, but also in the physiological process of ageing. Similarly, the end products of glycoxidation processes, advanced glycation end products (AGEs), are damaging compounds, a myriad of reports linking them to the development and progression of several age-associated chronic diseases. The aim of the present study was to analyze the relationships between zinc status, glycoxidative stress and insulin resistance (IR) in elderly subjects with type 2 diabetes mellitus (T2DM). A group of 52 non-smoking subjects ( 9 men and 43 women, aged 65-83 years) were enrolled in this cross-sectional study: 27 patients with T2DM, and 25 apparently healthy control subjects. Serum zinc (Zn) levels were assessed using a commercial kit based on an end-point colorimetric method, and serum AGEs were evaluated with a fluorimetric analytic procedure. The calculated glucose-to-zinc ratio (Gly/Zn), insulin-to-zinc ratio ( $\mathrm{Ins} / \mathrm{Zn}$ ) and insulin-zinc resistance index (HOMA-IR/Zn) were further used to study the associations between serum $\mathrm{Zn}$ levels, secretory function of $\beta$-pancreatic cells and AGEs. T2DM patients presented significantly higher serum insulin and $\mathrm{Zn}$ levels, as compared to the controls. We found a significant inverse correlation between $\mathrm{Zn}$ and AGEs, and a strong positive correlation
\end{abstract}

Correspondence to: Professor Daniela Grădinaru, Department of Biochemistry, Faculty of Pharmacy, 'Carol Davila' University of Medicine and Pharmacy, 6 Traian Vuia Street, 020956 Bucharest, Romania

E-mail: daniela.gradinaru@umfcd.ro

${ }^{*}$ Contributed equally

Key words: zinc, type 2 diabetes, ageing, advanced glycation end products, insulin resistance between AGEs and the Gly/Zn ratio, suggesting that both $\mathrm{Zn}$ and AGEs are biomarkers that could reflect the persistence of hyperglycemia. We identified new surrogate biomarkers useful for the assessment of glycemic control with great potential for the development of preventive and therapeutic strategies for elderly diabetics, based on the evaluation of serum $\mathrm{Zn}$ levels.

\section{Introduction}

Zinc $(\mathrm{Zn})$ is one of the most important micronutrients, whose deficiency has a great impact on biological, biochemical and immune functions (1). The direct link between insulin secretion and function, and $\mathrm{Zn}$ concentration was established around 1930, $\mathrm{Zn}$ being identified as one of the ions inducing insulin crystallization as well as its action; later on it was shown that $\mathrm{Zn}$ is essential in the pancreatic $\beta$-cell for processing and storage of insulin (2).

Recent literature data demonstrate that the dysregulation of $\mathrm{Zn}$ metabolism is associated with an increased risk of diabetes, as well as the impairment of the immune response, the latter being itself a component of diabetes mellitus (DM) etiopathology. Zn physiology in the $\beta$-cell depends on the function of ZnT8, a transporter encoded by the SLC30A8 gene, whose polymorphism is associated with high type 2 DM (T2DM) risk (3). Among all the trace elements studied, the plasma $\mathrm{Zn}$ concentration seems to be a useful biomarker of ageing since its concentration has been shown to slowly decline with age in many different studies $(4,5)$. At the same time, the intracellular concentration of metallothioneins (MTs), the main intracellular proteins involved in $\mathrm{Zn}$ homeostasis, was shown to increase during ageing and age-related diseases $(6,7)$. Zn deficiencies are reported in the course of ageing and in different diseases, such as DM, obesity, atherosclerosis, as well as rheumatoid arthritis or respiratory tract infections, among others (2,8-14). In fact, the age-related changes of trace element concentrations in biological fluids/tissues are not only attributed to a deficient nutritional status, but also to an intrinsic dysregulated homeostasis (1). 
At the systemic level, glycation and oxidative stress resulting from hyperglycemia and dyslipidemia lead to accelerated non-enzymatic modification of essential biomolecules, particularly of proteins (15-19). In particular, advanced glycation end products (AGEs) are represented by a heterogenous group of damaging compounds (e.g., pentosidine, carboxymethyllysine, and imidazolone) that are formed by nonenzymatic glycation of macromolecules; some of them have characteristic fluorescence, with the ability of crosslinking of protein and interacting with AGE-specific receptor RAGE (20-24). AGE accumulation in tissues reflects cumulative metabolic stress, or 'metabolic memory' rather than short-term glycemic control, thus pointing to protein tissue damage resulted from the effects of exposure to many cardiovascular disease (CVD) risk factors $(17,25-27)$.

Despite the numerous studies, there is still limited information concerning the relationships between $\mathrm{Zn}$ status, glycoxidative stress and IR in elderly with impaired glucose metabolism. The aim of this pilot study was to evaluate serum Zn levels in elderly subjects with T2DM and analyze the biological and clinical relevance of this biomarker in terms of glycoxidative stress, metabolic profile and pancreatic function.

\section{Patients and methods}

Subjects. A total of 52 non-smoking elderly subjects (9 men and 43 women, aged $65-83$ years, mean age: $70+5$ years) were enrolled in this cross-sectional study: a T2DM group composed of 27 known type 2 diabetic patients treated at 'Prof. Nicolae Paulescu' INMD clinic department (during September 2019), and 25 apparently healthy control subjects selected from the patients admitted at Ana Aslan National Institute of Gerontology and Geriatrics (during September 2019), with normal glucose levels and normal lipid profile, recruited from the outpatient clinical department. Subjects were included in the T2DM study group, according to the clinical and biochemical criteria of the American Diabetes Association (28). Diabetic patients were on different oral antidiabetic medication, mainly metformin and gliclazide. Exclusion criteria included acute infection, acute myocardial infarction, active liver disease or liver dysfunction, renal impairment, hematological and malignant overt diseases. Patients who were on insulin or taking any antioxidant or $\mathrm{Zn}$ supplements were also excluded.

Biochemical parameters. A single blood sample $(6 \mathrm{ml})$ was obtained from each subject after an 8-12 h overnight fasting. Serum samples were separated by centrifugation (3,000 x $\mathrm{g}$ for $10 \mathrm{~min}$ ) from whole blood for the assessment of metabolic profile: glucose, total cholesterol, high-density lipoprotein cholesterol (HDL-C), low-density lipoprotein cholesterol (LDL-C), blood urea nitrogen, creatinine and uric acid, using standard methods on a Cobas C311 Analyzer and Cobas Integra 400 Analyzer for HbA1C (Roche Diagnostics Ltd., Switzerland). Fasting insulin levels were assessed with a Sandwich ELISA based assay (EIA-2935, DRG Diagnostics). The inter-assay coefficient of variation (CV) was $6.0 \%$, and the intra-assay $\mathrm{CV}$ was $2.6 \%$. Insulin resistance (IR) was evaluated using the homeostasis model assessment-insulin resistance (HOMA-IR): fasting insulin (mU/1) $\mathrm{x}$ fasting glucose (mg/dl) divided by 405 (29). The serum AGEs were assessed by the level of AGE-associated autofluorescence recorded at $440 \mathrm{~nm}$ emission wavelength upon excitation at $350 \mathrm{~nm}$, as previously described (17). The measurements were performed on PerkinElmer Spectrofluorimeter (LS 50 B, PerkinElmer, Inc.). Fluorescence intensity was expressed in relative fluorescence units (RFU). The coefficient of variation $(\mathrm{CV})$ for replicate measurements was $<5 \%$. $\mathrm{Zn}$ assessment in serum samples was performed using a sensitive, standardized, simple and direct procedure (reagent kit MAK032, Sigma-Aldrich; Merck KGaA) which measures the colorimetric product $(560 \mathrm{~nm})$ resulting from the binding of $\mathrm{Zn}$ ions to a specific ligand, namely 5-Bromo-PAPS [2-(5-Bromo-2-pyridylazo)-5-[N-propyl-N-(3-sulfopropyl) amino] phenol disodium salt dihydrate]. Concentration of serum $\mathrm{Zn}$ was expressed in $\mathrm{mM} \mathrm{Zn}$ (mmol/l serum). The inter-assay and intra-assay coefficients of variation were 5.4 and $6.7 \%$, respectively. Biochemical and immunoenzymatic tests were performed on ChemWell 2190 Analyzer (Awareness Technology).

Indices of glycemic control and Zn status. The glucose-to-zinc ratio $(\mathrm{Gly} / \mathrm{Zn})$ was calculated using the fasting levels of serum glucose and $\mathrm{Zn}$, expressed both as $\mathrm{mmol} / \mathrm{l}$ serum. The insulin-to-zinc ratio (Ins/Zn) was calculated using the fasting levels of serum insulin (mU/l serum) and $\mathrm{Zn}$, expressed as mmol/l serum. The insulin-zinc resistance index (HOMA-IR/Zn) was calculated using HOMA-IR individual values and serum $\mathrm{Zn}$ levels.

Statistical analysis. We used the Statistical Package for Social Sciences software (SPSS, version 15, SPSS, Inc.). Comparison between the control group and T2DM group was performed using Student's unpaired t-test. Pearson's two-tailed bivariate correlations $(r)$ were performed to examine the associations of serum $\mathrm{Zn}$ and serum AGEs, with insulin, glucose, HOMA-IR, as well as the correlations between AGEs and Gly/Zn, Ins/Zn and HOMA-IR/Zn ratios. Both tests were two-sided and a $\mathrm{P}$-value $<0.05$ was considered statistically significant.

\section{Results}

The study population included 52 elderly subjects divided into two groups: a control group, with normal metabolic profile $(n=25)$, and a group with T2DM $(n=27)$. The biochemical and metabolic parameters measured in both groups are presented in Table I.

The mean values of biomarkers indicating the metabolic dysregulation and cardiovascular risk, namely the values of fasting glucose, insulin, and triglycerides were significantly higher in the T2DM group compared with the control group, whereas HDL-cholesterol levels were significantly lower. Insignificant differences between groups were observed for creatinine, whereas uric acid and blood urea nitrogen levels were slightly higher in the T2DM group. T2DM elderly patients were characterized by a significant hyperinsulinemia and subsequent significant increases in insulin resistance index HOMA-IR.

The glycoxidative stress was evaluated as an AGE biomarker as well as the traditional marker indicating the persistence of chronic hyperglycemia, HbA1C; both were 
Table I. Biochemical parameters in control and type 2 diabetes mellitus (T2DM) elderly subjects.

\begin{tabular}{|c|c|c|c|}
\hline Parameters & Control group $(n=25)$ & T2DM group $(n=27)$ & P-value \\
\hline Age, mean (years) & $69 \pm 5$ & $71 \pm 5$ & NS \\
\hline Fasting glucose (mg/dl) & $98.92 \pm 10.99$ & $152.65 \pm 30.23$ & $<0.001$ \\
\hline HbA1c (\%) & $5.88 \pm 0.62$ & $7.46 \pm 0.85$ & $<0.001$ \\
\hline Fasting insulin $(\mu \mathrm{U} / \mathrm{ml})$ & $4.45 \pm 1.21$ & $11.49 \pm 4.31$ & $<0.001$ \\
\hline HOMA-IR & $1.10 \pm 0.36$ & $4.26 \pm 1.57$ & $<0.001$ \\
\hline Total cholesterol (mg/dl) & $180.67 \pm 32.80$ & $198.96 \pm 33.94$ & NS \\
\hline Triglycerides (mg/dl) & $109.88 \pm 69.28$ & $167.69 \pm 64.52$ & $<0.01$ \\
\hline HDL-cholesterol (mg/dl) & $55.83 \pm 14.73$ & $43.73 \pm 11.58$ & $<0.01$ \\
\hline LDL-cholesterol (mg/dl) & $103.83 \pm 36.83$ & $119.92 \pm 30.86$ & NS \\
\hline Uric acid (mg/dl) & $4.42 \pm 1.67$ & $5.47 \pm 1.62$ & $<0.05$ \\
\hline Creatinine (mg/dl) & $0.86 \pm 0.16$ & $0.92 \pm 0.16$ & NS \\
\hline Blood urea nitrogen (mg/dl) & $38.79 \pm 13.50$ & $43.27 \pm 15.88$ & $<0.01$ \\
\hline AGEs (RFU) & $103.84 \pm 13.73$ & $120.74 \pm 21.47$ & $<0.01$ \\
\hline Serum zinc (mmol/l) & $14.11 \pm 2.06$ & $15.71 \pm 1.71$ & $<0.01$ \\
\hline
\end{tabular}

Values are expressed as mean $\pm \mathrm{SD}$; $\mathrm{n}$, number of subjects. $\mathrm{P}<0.05$ indicates statistical significance when compared to the control group; NS, not significant; HOMA-IR, homeostasis model assessment of insulin resistance; AGEs, advanced glycation end products.

significantly higher in elderly subjects with T2DM vs. the control group.

Serum Zn levels were significantly $(\mathrm{P}<0.01)$ increased in the T2DM group as compared to the control subjects. The mean $\mathrm{Zn}$ value in the T2DM group was $15.7 \mathrm{mmol} / \mathrm{l}$ (range: 12.03-19.12 $\mathrm{mmol} / \mathrm{l}$ ), whereas in the control group the average serum $\mathrm{Zn}$ concentration was $14.11 \mathrm{mmol} / \mathrm{l}$ (range: 9.89-17.31 mmol/1).

Overall, elderly subjects with T2DM presented significantly higher serum insulin and Zn levels, as well as HOMA-IR values, as compared to the control. Therefore, the calculated Gly/Zn, Ins/Zn and insulin-zinc resistance index (HOMA-IR/Zn) were further used as surrogate biomarkers to explore the relationships between serum $\mathrm{Zn}$ levels, metabolic profile, secretory functions of $\beta$-pancreatic cells and glycoxidative stress. All of these three metabolic indices illustrative of the glycemic control and Zn status displayed significantly higher values in T2DM patients than in the control subjects (Fig. 1).

In T2DM patients we aimed to explore the relationships between the advanced glycation of proteins (AGEs), Zn systemic status and glucose homeostasis. The correlation analysis was carried out between levels of AGEs and serum $\mathrm{Zn}$ levels, Gly/Zn ratio, Ins/Zn ratio and HOMA-IR/Zn ratio. Statistical significance of the Pearson's correlation coefficients was different among the four study biomarkers as presented in Fig. 2.

AGEs values showed a significant negative correlation ( $\mathrm{r}=-0.454 ; \mathrm{P}<0.02$ ) with $\mathrm{Zn}$ serum levels and a strong positive association $(\mathrm{r}=0.767 ; \mathrm{P}<0.001)$ with the $\mathrm{Gly} / \mathrm{Zn}$ ratio (Fig. 2A and B). Regarding the association between AGEs and $\beta$-cell secretory function, the correlation analysis identified significant negative relationships between AGEs and insulin $(\mathrm{r}=-0.459 ; \mathrm{P}<0.02)$ and significantly higher between AGEs and Ins/Zn ratio ( $\mathrm{r}=-0.523 ; \mathrm{P}<0.01)$ (Fig. $2 \mathrm{C})$. Despite the elevated

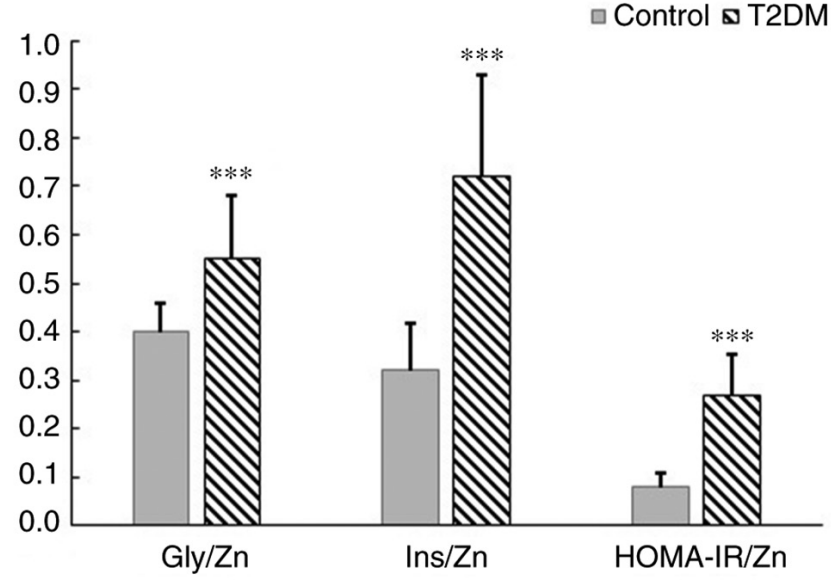

Figure 1. Differences between control $(n=25)$ and type 2 diabetes (T2DM) $(n=27)$ elderly subjects in the calculated glucose-to-zinc ratio (Gly/Zn), insulin-to-zinc ratio (Ins/Zn) and insulin-zinc resistance index (HOMA-IR/Zn), used as surrogate biomarkers to study the relationships between serum zinc levels, secretory function of $\beta$-pancreatic cells and glycoxidative stress. Values are expressed as mean \pm SD; $n$, number of subjects. Statistical significance when comparing against the control group $\left({ }^{* * * *} \mathrm{P}<0.001\right)$.

levels of circulating AGEs found out in hyperglycemic and insulin-resistant subjects, the association with HOMA-IR was not significant ( $\mathrm{r}=-0.145$; NS), whereas the correlation between AGEs and HOMA-IR/Zn ratio was significant ( $\mathrm{r}=-0.382$; $\mathrm{P}<0.05$ ) (Fig. 2D).

\section{Discussion}

The results of the present showed that elderly diabetic patients had significantly higher levels of serum $\mathrm{Zn}$ in comparison with the control group subjects. These results are in agreement with a recent study conducted in postmenopausal women 

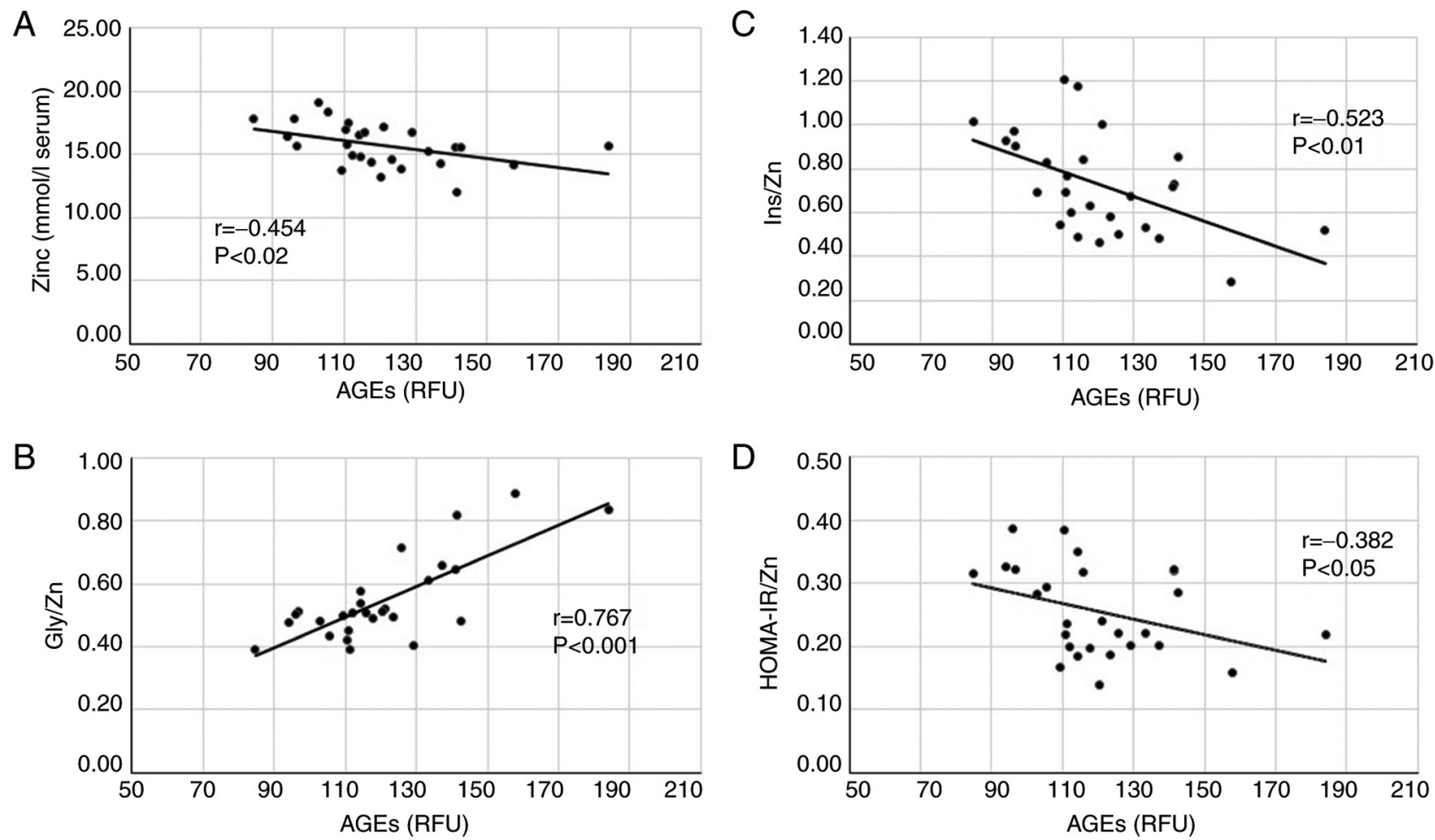

Figure 2. Pearson's correlation between serum advanced glycation end products (AGEs) and serum zinc (Zn) levels (A) glucose-to-zinc ratio (Gly/Zn) (B) insulin-to-zinc ratio (Ins/Zn) (C) and insulin-zinc resistance index (HOMA-IR/Zn) (D) in the type 2 diabetes group (T2DM) (n=27).

with diabetes, characterized by significantly higher Zn levels as compared to the controls (30). However, these data are in contrast with many studies, conducted mainly on adult population, in which T2DM individuals displayed a reduced $\mathrm{Zn}$ status correlated with a poor glycemic control (31-33). In a recent systematic review and meta-analysis, Yin et al showed that the serum $\mathrm{Zn}$ concentration was lower in polycystic ovary syndrome (PCOS) women with IR than in healthy women. However, there was no significant difference in serum $\mathrm{Zn}$ concentration when compared between healthy control and PCOS women without IR (34).

Understanding the complex role of $\mathrm{Zn}$ in glucose homeostasis and subsequent IR and T2DM is at present limited by controversial findings in the literature and the absence of 'gold standard' animal models that could help elucidate the role of $\mathrm{Zn}$ and $\mathrm{Zn}$ transporters (35). It is acknowledged that, at the pancreatic level, $\mathrm{Zn}$ participates in mechanisms involving insulin secretion and action, as a catalytic cofactor for carboxypeptidase $\mathrm{H}$, an enzyme which catalyzes the conversion from proinsulin into insulin. Zn also promotes phosphorylation of insulin receptor by enhancing glucose transport into cells (36). Previous studies have demonstrated the role of higher serum concentration of $\mathrm{Zn}$ in improving insulin sensitivity in T2DM (37). Regarding the roles of $\mathrm{Zn}$ in blood insulin levels, recent experimental studies conducted on mice with $\beta$-cell-specific genetic deficiency for ZnT8 transporter found an enhanced insulin secretion but also an enhanced insulin clearance in the liver, due to reduced $\mathrm{Zn}$ secretion from these cells (38).

In the present study, we also pointed out a significant inverse correlation between $\mathrm{Zn}$ levels and AGEs, and a strong positive correlation between the Gly/Zn ratios and AGEs, suggesting that both $\mathrm{Zn}$ and AGEs are biomarkers that could reflect the persistence of hyperglycemia. These results could also suggest that $\mathrm{Zn}$ could exert at the systemic level some anti-glycation actions, as confirmed in studies conducted in vitro (39). Therefore, we also made an attempt to 'normalize' the traditional indices of $\beta$-cells secretory function and IR to serum $\mathrm{Zn}$ levels and obtain new surrogate biomarkers, namely Ins/Zn and HOMA-IR/Zn ratios, and study their relationships with AGEs. In the elderly diabetic subjects, Ins/Zn and HOMA-IR/Zn ratios were significantly inversely correlated with the glycoxidative stress evaluated as AGEs.

The main limitation of the study was the reduced number of subjects and the absence of evaluation of proteins involved in $\mathrm{Zn}$ metabolism such as $\mathrm{Zn}-\alpha 2$-glycoprotein and Zn-induced metallothionein, that could help to elucidate the mechanisms involved in the interplay between pancreatic $\beta$-cells/Zn/glycoxidative stress.

In the present study, we propose, for the first time, the evaluation of these new $\mathrm{Zn}$ status surrogate biomarkers involved in glucose homeostasis, as numerous clinical markers and quotients are routinely used to evaluate the metabolic impairment. Similarly, a recent study on patients with polycystic ovary syndrome (PCOS), T2DM and impaired glucose tolerance (IGT) identified the natural logarithm of Zn- $\alpha 2$-glycoprotein/HOMA-IR as a better predictor of insulin sensitivity than the product of triglycerides and glucose and the other lipid ratios (40). Therefore, we evaluated the biochemical and clinical relevance serum AGEs and the newly introduced ratios of Ins/Zn and HOMA-IR/Zn, which could be useful 
in clinical practice in the diagnosis of IR associated with $\mathrm{Zn}$ deficiency and oxidative stress.

In conclusion, in the present study conducted on elderly diabetic subjects, we identified new significant associations between $\mathrm{Zn}$ status, glycoxidative stress, pancreatic $\beta$-cell impairment and IR. Similar approaches could help in the future for the development of preventive strategies for personalized nutrition and treatment, that would increase the longevity and quality of life of patients with diabetes.

\section{Acknowledgements}

Not applicable.

\section{Funding}

The experiments carried out in this study and the publication of the article were supported by regular financial resources provided by 'Carol Davila' University of Medicine and Pharmacy within the general framework for the development of scientific research collaboration.

\section{Availability of data and materials}

The datasets used and/or analyzed during the current study are available from the corresponding author.

\section{Authors' contributions}

DG and DM conceived and designed the study, contributed to the experiments and drafted the manuscript. AU, GN and CMP performed all the experiments and the statistical analysis. RDM performed the clinical data analysis. CMP, CIT and RDM contributed to the conception and designed the study. All the authors have read and approved the final manuscript for publication.

\section{Ethics approval and consent to participate}

The study protocol was approved by 'Nicolae Paulescu' Institute of Diabetes, Metabolic and Nutrition Diseases (no. 10403/2019) and 'Ana Aslan' National Institute of Gerontology and Geriatrics (no. 12660/2019) Ethics Committees and all subjects provided their informed consent before participation.

\section{Patient consent for publication}

Not applicable.

\section{Competing interests}

The authors declare that they have no competing interests.

\section{References}

1. Mocchegiani E, Romeo J, Malavolta M, Costarelli L, Giacconi R, Diaz LE and Marcos A: Zinc: Dietary intake and impact of supplementation on immune function in elderly. Age (Dordr) 35: 839-860, 2013.

2. Maret W: Zinc in pancreatic islet biology, insulin sensitivity, and diabetes. Prev Nutr Food Sci 22: 1-8, 2017.
3. Huang Q, Du J, Merriman C and Gong Z: Genetic, functional, and immunological study of ZnT8 in diabetes. Int J Endocrinol 2019: 1524905,2019

4. Giacconi R, Costarelli L, Piacenza F, Basso A, Rink L, Mariani E, Fulop T, Dedoussis G, Herbein G, Provinciali M, et al: Main biomarkers associated with age-related plasma zinc decrease and copper/zinc ratio in healthy elderly from ZincAge study. Eur J Nutr 56: 2457-2466, 2017.

5. Malavolta M, Piacenza F, Basso A, Giacconi R, Costarelli L and Mocchegiani E: Serum copper to zinc ratio: Relationship with aging and health status. Mech Ageing Dev 151: 93-100, 2015.

6. Giacconi R, Costarelli L, Piacenza F, Basso A, Burkle A, Moreno-Villanueva M, Grune T, Weber D, Stuetz W, Gonos ES, et al: Zinc-induced metallothionein in centenarian offspring from a large European population: The MARK-AGE Project. J Gerontol A Biol Sci Med Sci 73: 745-753, 2018.

7. Giacconi R, Cai L, Costarelli L, Cardelli M, Malavolta M, Piacenza F and Provinciali M: Implications of impaired zinc homeostasis in diabetic cardiomyopathy and nephropathy. Biofactors 43: 770-784, 2017.

8. Kloubert V and Rink L: Zinc as a micronutrient and its preventive role of oxidative damage in cells. Food Funct 6: 3195-3204, 2015.

9. Skalny AV, Rink L, Ajsuvakova OP, Aschner M, Gritsenko VA, Alekseenko SI, Svistunov AA, Petrakis D, Spandidos DA, Aaseth J, et al: Zinc and respiratory tract infections: Perspectives for COVID19 (Review). Int J Mol Med 46: 17-26, 2020.

10. Rios-Lugo MJ, Madrigal-Arellano C, Gaytán-Hernández D, Hernández-Mendoza H and Romero-Guzmán ET: Association of serum zinc levels in overweight and obesity. Biol Trace Elem Res 198: 51-57, 2020.

11. Rajaee E, Mowla K, Ghorbani A, Dargahi-Malamir M, Zarei M and Rahimikhah FA: The relationship between serum zinc levels and rheumatoid arthritis activity. Front Biol 13: 51-55, 2018.

12. Choi S, Liu X and Pan Z: Zinc deficiency and cellular oxidative stress: Prognostic implications in cardiovascular diseases. Acta Pharmacol Sin 39: 1120-1132, 2018.

13. Farhood HB, Balas M, Gradinaru D, Margina D and Dinischiotu A: Hepatoprotective effects of chlorogenic acid under hyperglycemic conditions. Rom Biotechnol Lett 24: 301-307, 2019.

14. Farhood HB, Balas MR, Gradinaru D, Margina D and Dinischiotu A: Effects of chlorogenic acid on the liver cell metabolism under high glucose conditions. Rom Biotechnol Lett 24: 883-892, 2019.

15. Ungurianu A, Seremet O, Gradinaru D, Ionescu-Tirgoviste C, Margina D and Danciulescu Miulescu R: Spectrophotometric versus spectrofluorometric assessment in the study of the relationships between lipid peroxidation and metabolic dysregulation. Chem Biol Drug Des 93: 1026-1035, 2019.

16. Ungurianu A, Margina D, Gradinaru D, Bacanu C, Ilie M, Tsitsimpikou C, Tsarouhas K, Spandidos DA and Tsatsakis AM: Lipoprotein redox status evaluation as a marker of cardiovascular disease risk in patients with inflammatory disease. Mol Med Rep 15: 256-262, 2017.

17. Gradinaru D, Borsa C, Ionescu C and Margina D: Advanced oxidative and glycoxidative protein damage markers in the elderly with type 2 diabetes. J Proteomics 92: 313-322, 2013.

18. Margina D, Ilie M and Gradinaru D: Quercetin and epigallocatechin gallate induce in vitro a dose-dependent stiffening and hyperpolarizing effect on the cell membrane of human mononuclear blood cells. Int J Mol Sci 13: 4839-4859, 2012.

19. Gradinaru D, Margina D and Borsa C: In vitro studies regarding the antioxidant effects of procaine, Gerovital $\mathrm{H} 3$ and Aslavital. Rev Roum Chim 54: 761-766, 2009.

20. Gradinaru D, Khaddour H, Margina D, Ungurianu A, Borsa C, Ionescu C, Prada GI, Usher J and Elshimali Y: Insulin-leptin axis, cardiometabolic risk and oxidative stress in elderly with metabolic syndrome. Exp Clin Endocrinol Diabetes: Feb 8, 2018. (Epub ahead of print). doi: 10.1055/s-0043-123825 2018.

21. Gradinaru D, Margina D, Borsa C, Ionescu C, Ilie M, Costache M, Dinischiotu A and Prada GI: Adiponectin: Possible link between metabolic stress and oxidative stress in the elderly. Aging Clin Exp Res 29: 621-629, 2017.

22. Gradinaru D, Margina D, Ilie M, Borsa C, Ionescu C and Prada GI: Correlation between erythropoietin serum levels and erythrocyte susceptibility to lipid peroxidation in elderly with type 2 diabetes. Acta Physiol Hung 102: 400-408, 2015. 
23. Borsa C, Gradinaru D, Margina D, Prada GI and Pena C: Receptor of advanced glycation end products and cardiovascular risk in elderly with type 2 diabetes mellitus. J Biol Res 90: 81-86, 2017.

24. Margina D, Gradinaru D and Mitrea N: Development of a potentiometric method for the evaluation of redox status in human serum. Rev Roum Chim 54: 45-48, 2009.

25. Zanfirescu A, Cristea AN, Nitulescu GM, Velescu BS and Gradinaru D: Chronic monosodium glutamate administration induced hyperalgesia in mice. Nutrients 10: 1, 2017.

26. Zanfirescu A, Ungurianu A, Tsatsakis AM, Nitulescu GM Kouretas D, Veskoukis A, Tsoukalas D, Engin AB, Aschner M and Margina D: A review of the alleged health hazards of monosodium glutamate. Compr Rev Food Sci Food Saf 18: 1111-1134, 2019.

27. Ungurianu A, Seremet O, GagniucE, Olaru OT, Gutu C, GradinaruD, Ionescu-Tirgoviste C, Margina D and Danciulescu-Miulescu R: Preclinical and clinical results regarding the effects of a plant-based antidiabetic formulation versus well established antidiabetic molecules. Pharmacol Res 150: 104522, 2019.

28. American Diabetes Association: Improving care and promoting health in populations: Standards of medical care in diabetes-2020. Diabetes Care 43 (Suppl 1): S7-S13, 2020.

29. Matthews DR, Hosker JP, Rudenski AS, Naylor BA, Treacher DF and Turner RC: Homeostasis model assessment: Insulin resistance and beta-cell function from fasting plasma glucose and insulin concentrations in man. Diabetologia 28: 412-419, 1985.

30. Skalnaya MG, Skalny AV and Tinkov AA: Serum copper, zinc, and iron levels, and markers of carbohydrate metabolism in postmenopausal women with prediabetes and type 2 diabetes mellitus. J Trace Elem Med Biol 43: 46-51, 2017.

31. Bandeira VDS, Pires LV, Hashimoto LL, Alencar LL, Almondes KGS, Lottenberg SA and Cozzolino SMF: Association of reduced zinc status with poor glycemic control in individuals with type 2 diabetes mellitus. J Trace Elem Med Biol 44: 132-136, 2017.
32. Sobczak AIS, Stefanowicz F, Pitt SJ, Ajjan RA and Stewart AJ: Total plasma magnesium, zinc, copper and selenium concentrations in type-I and type-II diabetes. Biometals 32: 123-138, 2019.

33. Farooq DM, Alamri AF, Alwhahabi BK, Metwally AM and Kareem KA: The status of zinc in type 2 diabetic patients and its association with glycemic control. J Family Community Med 27: 29-36, 2020.

34. Yin J, Hong X, Ma J, Bu Y and Liu R: Serum trace elements in patients with polycystic ovary syndrome: A systematic review and meta-analysis. Front Endocrinol (Lausanne) 11: 572384 , 2020.

35. Pearsey HM, Henson J, Sargeant JA, Davies MJ, Khunti K, Suzuki T, Bowden-Davies KA, Cuthbertson DJ and Yates TE: Zinc-alpha2-glycoprotein, dysglycaemia and insulin resistance: A systematic review and meta-analysis. Rev Endocr Metab Disord 21: 569-575, 2020.

36. Capdor J, Foster M, Petocz P and Samman S: Zinc and glycemic control: A meta-analysis of randomised placebo controlled supplementation trials in humans. J Trace Elem Med Biol 27: 137-142, 2013.

37. Vashum KP, McEvoy M, Milton AH, Islam MR, Hancock S and Attia J: Is serum zinc associated with pancreatic beta cell function and insulin sensitivity in pre-diabetic and normal individuals? Findings from the Hunter Community Study. PLoS One 9: e83944, 2014.

38. Watada $\mathrm{H}$ and Tamura Y: Impaired insulin clearance as a cause rather than a consequence of insulin resistance. J Diabetes Investig 8: 723-725, 2017.

39. Kheirouri S, Alizadeh $M$ and Maleki V: Zinc against advanced glycation end products. Clin Exp Pharmacol Physiol 45: 491-498, 2018.

40. Qu C, Zhou X, Yang G, Li L, Liu H and Liang Z: The natural logarithm of zinc- $\alpha 2$-glycoprotein/HOMA-IR is a better predictor of insulin sensitivity than the product of triglycerides and glucose and the other lipid ratios. Cytokine 79: 96-102, 2016. 